\title{
On Equitable Irregular graphs
}

\author{
P. Sanakara Narayanan, S. Saravanakumar
}

Abstract: An k-edge-weighting of a graph $G=(V, E)$ is a map $\varphi: E(G) \rightarrow\{1,2,3, \ldots k\}$, \}where $k \geq 1$ is an integer. Denote $S_{\varphi}(v)$ is the sum of edge-weights appearing on the edges incident at the vertex $v$ under $\varphi$. An k-edge -weighting of $G$ is equitable irregular if $\left|S_{\varphi}(u)-S_{\varphi}(v)\right| \leq 1$, for every pair of adjacent vertices $u$ and $v$ in $G$. The equitable irregular strength $S_{e}(G)$ of an equitable irregular graph $G$ is the smallest positive integer $k$ such that there is a $k$-edge weighting of $G$. In this paper, we discuss the equitable irregular edge-weighting for some classes of graphs.

Keywords :Edge-weighting, equitable irregular graphs.

\section{INTRODUCTION}

A graph labeling is an assignment of integers to the vertices or edges or both with respect to some conditions. A detailed survey of graph labeling is given by Gallian [4]. This paper considering an edge labeling of graphs. For our convenience, we call the term edge - weighting instead of edge - labeling. An k-edge weighting is a $\operatorname{map} \varphi: E(G) \rightarrow\{1,2, \ldots, k\}$, where $k \in N \mathrm{~N}$ is equitable irregular if $\left|S_{\varphi}(u)-S_{\varphi}(v)\right| \leq$ 1 for every pair of adjacent vertices $u$ and $v$ in $G$, where $S_{\varphi}(x)$ is the sum of the edge - weights presenting on the edges incident at the vertex $x$. A graph admits such a labeling is called an equitable irregular. This notion was introduced by I. Sahul Hamid and S. Ashok Kumar in [3]. In that paper, the authors were discussed some properties of equitable irregular graphs and provided some classes of equitable irregular graphs along with its strength. In this paper, we extend the study of this parameter by proving closed helm graphs, windmill graphs, flower graphs, quadrilateral snake and double quadrilateral snake graphs are equitable irregular. Moreover, we determine the exact value of the strength for each of these classes of graphs. For this, we need the following theorem which is proved in [3].

Theorem 1.1. If $\mathrm{G}$ is equitable irregular graph, $\operatorname{then} S_{e}(G) \geq$ $\left\lceil\frac{\Delta(G)-2}{\mu(G)-1}\right\rceil$, where $\mu(G)=\min \left\{\mu_{x}: x \in V(G)\right\}$,

$\operatorname{deg} x=\Delta(G)$ and $\mu(x)=\min \{\operatorname{deg} y: x y \in E(G)\}$.

\section{MAIN RESULTS}

In this section, we prove that the closed helm graphs, windmill graphs, flower graphs, quadrilateral and double quadrilateral graphs are equitable irregular. Moreover, we compute the irregularity strength of these families.

Definition 2.1. A closed helmCH$H_{n}$ is the graph obtained by taking a helm $H_{n}$ and adding edges between the pendant vertices to form a cycle.

\footnotetext{
Revised Manuscript Received on December 29, 2019.

* Correspondence Author

P. Sankara Narayanan, Department of Mathematics, Kalasalingam Academy of Research and Education, Krishnankoil, India.

Email: sankarmath4@gmail.com

*S. Saravanakumar, Department of Mathematics, KalasalingamAcademy of Research and Education, Krishnankoil, India.

Email: alg.ssk@gmail.com
}

Theorem 2.2. For all $n$, the graph $\mathrm{CH}_{n}$ is equitable irregular and $S_{e}\left(\mathrm{CH}_{n}\right)=\left\lceil\frac{n-2}{3}\right\rceil$.

Proof. Let $\mathrm{V}\left(\mathrm{CH}_{n}\right)=\left\{v_{0}, v_{1}, v_{1}{ }^{\prime}, v_{2}, v_{2}{ }^{\prime}, \ldots, v_{n}, v_{n}{ }^{\prime}\right\}$ and $E\left(C H_{n}\right)=\left\{v_{0} v_{i}: 1 \leq i \leq n\right\} \cup\left\{v_{i} v_{i}^{\prime}: 1 \leq i \leq n\right\} \cup$

$\left\{v_{i} v_{i+1}: 1 \leq i \leq n-1\right\} \cup\left\{v_{i} v_{i+1}{ }^{\prime}: 1 \leq i \leq n-1\right\} \cup$

$\left\{v_{1} v_{n}, v_{1}^{\prime} v_{n}^{\prime}\right.$. Define an edge-weighting $\varphi$ of $C H_{n}$ as follows.

Let $\varphi\left(v_{0} v_{i}\right)=1$ for all $1 \leq i \leq n$ and assign $\left\lceil\frac{n-2}{3}\right\rceil$ forall the remaining edges of $\mathrm{CH}_{n}$. (For the graph $\mathrm{CH}_{n}$, the edge-weighting $\varphi$ is illustrated in Figure 1).

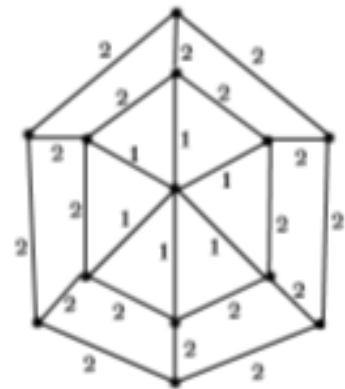

Figure 1: The graph $\mathrm{CH}_{6}$ and its irregular edge-weighting

Then $S_{\varphi}\left(v_{0}\right)=n$ and for all $1 \leq i \leq n$, we have $S_{\varphi}\left(v_{0}\right)=\varphi\left(v_{0} v_{i}\right)+\varphi\left(v_{i} v_{i}^{\prime}\right)+\varphi\left(v_{i} v_{i+1}\right)+\varphi\left(v_{i} v_{i-1}\right)$

$$
= \begin{cases}n+1 & \text { if } n \equiv 0(\bmod 3) \\ n & \text { if } n \equiv 1(\bmod 3) \\ n-1 & \text { if } n \equiv 2(\bmod 3)\end{cases}
$$

Further, $S_{\varphi}\left(v_{i}{ }^{\prime}\right)=\varphi\left(v_{i} v_{i}{ }^{\prime}\right)+\varphi\left(v_{i}{ }^{\prime} v_{i+1}{ }^{\prime}\right)+\varphi\left(v_{i}{ }^{\prime} v_{i-1}{ }^{\prime}\right)$

$$
= \begin{cases}n & \text { if } n \equiv 0(\bmod 3) \\ n-1 & \text { if } n \equiv 1(\bmod 3) \\ n-2 & \text { if } n \equiv 2(\bmod 3)\end{cases}
$$

It is clear that, the difference of the weights of any two adjacent vertices of $\mathrm{CH}_{n}$ under $\varphi$ is at most 1 . Hence $\mathrm{CH}_{n}$ is equitable irregular and so $S_{e}\left(\mathrm{CH}_{n}\right) \leq\left\lceil\frac{n-2}{3}\right\rceil$. Since $\mu\left(\mathrm{CH}_{n}\right)=$ 4 and $\Delta\left(C_{n}\right)=n$, it follows by Theorem 1.1, we get $S_{e}\left(C_{n}\right) \geq\left\lceil\frac{n-2}{3}\right\rceil$. Thus $S_{e}\left(C_{n}\right)=\left\lceil\frac{n-2}{3}\right\rceil$.

Definition 2.3. The windmill $\operatorname{graph} W_{n}{ }^{(m)}$ is the graph obtained by taking $m$-copies of the complete graph $K_{n}$ with a vertex in common.

Theorem 2.4. For all $n$ and $m$, the windmill $\operatorname{graph} W_{n}{ }^{(m)}$ is equitable irregular.

Proof:Let $V\left(W_{n}{ }^{(m)}\right)=\left\{v_{0}, v_{11}, v_{12}, v_{13}, \ldots, v_{1(n-1)}, v_{21}, v_{22}\right.$,

$\left.v_{23}, \ldots, v_{2(n-1)} \ldots, v_{m 1}, v_{m 2}, v_{m 3}, \ldots, v_{m(n-1)}\right\}$

and $E\left(W_{n}{ }^{(m)}\right)=\left\{E\left(G_{i}\right): 1 \leq i \leq m\right\} \cup$

$\left\{v_{0} v_{i j}: 1 \leq i \leq n, 1 \leq j \leq n-1\right\}$,

$V\left(G_{i}\right)=$

$\left\{v_{i 1}, v_{i 2}, v_{i 3}, \ldots, v_{i(n-1)}: 1 \leq i \leq\right.$ $m\}$. 


\section{On Equitable Irregular graphs}

We now assign the weights to the edges of $W_{n}{ }^{(m)}$ as follows. Let $\varphi\left(v_{0} v_{i j}\right)=1$ for all $i=1,2,3 \ldots m$ and $j=$ $1,2,3, \ldots, n-1$. Further assign the weights $\left\lceil\frac{m n-m-2}{n-2}\right\rceil$ to all the remaining edges of $W_{n}{ }^{(m)}$. (For the graph $W_{4}{ }^{(3)}$ the edges-weighting $\varphi$ is illustrated in Figure 2). Then edge weighting of $W_{n}^{(m)}$ as follows.

For all $1 \leq i \leq m$ and $1 \leq i \leq n-1$, we have $S_{\varphi}\left(v_{i j}\right)=$ $1+(n-2)\left\lceil\frac{m n-m-2}{n-2}\right\rceil$ and $S_{\varphi}\left(v_{0}\right)=m(n-1)$,

$$
S_{\varphi}\left(v_{n m}\right)=\left\{\begin{array}{lr}
m(n-1)+1 & \text { if } n, m \text { are odd } \\
m(n-1) & \text { ifm is odd, } n \text { is even } \\
m(n-1)-1 & \text { otherwise }
\end{array}\right.
$$

Clearly for any two adjacent vertices of $W_{n}{ }^{(m)}$, their weights are differ by at most one and hence $W_{n}{ }^{(m)}$ is equitable irregular. Thus $S_{e}\left(W_{n}{ }^{(m)}\right) \leq\left\lceil\frac{m n-m-2}{n-2}\right\rceil$.

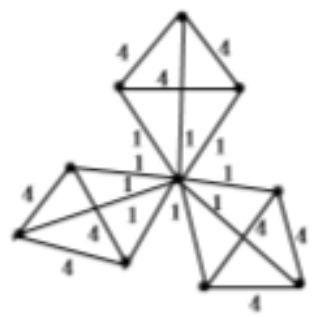

Figure 2: The graph $W_{f}^{(3)}$ and its irregualr edge weighting

Since $\Delta\left(W_{n}^{(m)}\right)=(n-1) m \quad$ and $\mu\left(W_{n}{ }^{(m)}\right)=n-1$, it follows from Theorem 1.1 we get $S_{e}\left(W_{n}{ }^{(m)}\right) \geq\left\lceil\frac{m n-m-2}{n-2}\right\rceil$. Thus $S_{e}\left(W_{n}{ }^{(m)}\right)=\left\lceil\frac{m n-m-2}{n-2}\right\rceil$.

Definition 2.5. A flower rraphFl$_{n}$ is the graph obtained from a closed helm by joining each pendant vertex of the helm toits center vertex.

Theorem 2.6. The flower graph $F l_{n}$ is equitable irregular for all $n$ and $S_{e}\left(F l_{n}\right)=\left\lfloor\frac{2 n}{3}\right\rfloor$.

Proof: Let $V\left(F l_{n}\right)=\left\{v_{0}, v_{1}, v_{1}{ }^{\prime}, v_{2}, v_{2}{ }^{\prime}, \ldots, v_{n}, v_{n}{ }^{\prime}\right\}$ and $E\left(F l_{n}\right)=\left\{v_{0} v_{i}: 1 \leq i \leq n\right\} \cup\left\{v_{i} v_{i}^{\prime}: 1 \leq i \leq n\right\} \cup$

$\left\{v_{i} v_{i+1}: 1 \leq i \leq n-1\right\} \cup\left\{v_{i} v_{i+1}: 1 \leq i \leq n-1\right\} \cup$

$\left\{v_{0} v_{i}^{\prime}: 1 \leq i \leq n\right\} \cup\left\{v_{1} v_{n}, v_{1}^{\prime} v_{0}^{\prime}\right\}$.

Now, let us define an edge-weighting $\varphi$ of $F l_{n}$ as follows.

For all $1 \leq i \leq n$, let $\varphi\left(v_{0} v_{i}\right)=\varphi\left(v_{0} v_{i}^{\prime}\right)=1$ and assign $\left\lfloor\frac{2 n}{3}\right\rfloor$ to all remaining edges of $F l_{n}$. Then $S_{\varphi}\left(v_{0}\right)=2 n$ and for all $1 \leq i \leq n$, we have

$S_{\varphi}\left(v_{i}\right)=S_{\varphi}\left(v_{i}{ }^{\prime}\right)== \begin{cases}2 n & \text { if } n \equiv 2(\bmod 3) \\ 2 n+1 & \text { if } n \equiv 0(\bmod 3) \\ 2 n-1 & \text { if } n \equiv 1(\bmod 3)\end{cases}$

One can easily verify that the difference of the weights of any two adjacent vertices of $F l_{n}$ is at most 1 and hence $F l_{n}$ is equitable irregular. Thus $S_{e}\left(F l_{n}\right) \leq\left\lfloor\frac{2 n}{3}\right\rfloor$. Here $\mu\left(F l_{n}\right)=4$ and $\Delta\left(F l_{n}\right)=2 \mathrm{n}$, we get $S_{e}\left(F l_{n}\right) \geq\left\lfloor\frac{2 n}{3}\right\rfloor$ by Theorem1.1. Hence $S_{e}\left(F l_{n}\right)=\left\lfloor\frac{2 n}{3}\right\rfloor$.

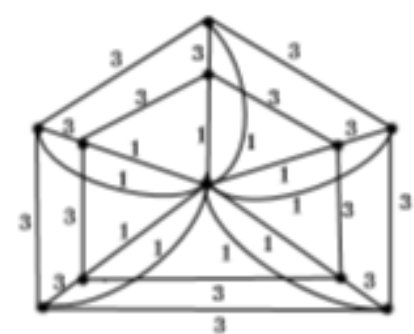

Figure 3: The graph $\mathrm{Fls}$ and its irrepular edge weighting

Definition 2.7. The Lotus inside a circle $L C_{n}$ is a graph obtained from the cycle $C_{n}:\left(u_{1}, u_{2}, \ldots, u_{m}, u_{1}\right)$ and star $K_{1, n}$ with center vertex $v_{0}$ and end vertices $v_{1}, v_{2}, \ldots, v_{n}$ by joining each $v_{i}$ to $u_{i}$ and $u_{i+1}(\bmod n)$.

Theorem 2.8. The Lotus inside a circle graph $L C_{n}$ is equitable irregular for all $n$ and $\mathrm{S}_{\mathrm{e}}\left(\left(L C_{n}\right)=\left\lfloor\frac{n-1}{2}\right\rfloor\right.$.

Proof: Let $V\left(L C_{n}\right)=\left\{v_{0}, v_{1}, u_{1}, v_{2}, u_{2}, \ldots, v_{n}, u_{n}\right\}$ and $E\left(L C_{n}\right)=\left\{v_{0} v_{i}: 1 \leq i \leq n\right\} \cup\left\{v_{i} u_{i}: 1 \leq i \leq n\right\} \cup$

$\left\{v_{i} u_{i+1}: 1 \leq i \leq n-1\right\} \cup\left\{v_{n} u_{1}\right\} \cup\left\{u_{i} u_{i+1}{ }^{\prime}: 1 \leq i \leq n-\right.$ 1Uunu1.

Now, let us define an edge-weighting $\varphi$ of $L C_{n}$ as follows. $\varphi\left(v_{0} v_{i}\right)=\varphi\left(u_{i} u_{i+1}\right)=\varphi\left(u_{1} u_{n}\right)=1$ for all $1 \leq i \leq$ $n$ and assign $\left\lfloor\frac{n-1}{2}\right\rfloor$ to all the remaining edges of $L C_{n}$. (Forthe graph $L C_{5}$, the equitable irregular edge-weighting $\varphi$ is illustrated in Figure 4.)

Clearly, $S_{\varphi}\left(v_{0}\right)=S_{\varphi}\left(u_{n}\right)=n$ and

$$
S_{\varphi}\left(v_{n}\right)= \begin{cases}n & \text { if } n \text { is odd } \\ n-1 & \text { if } n \text { is even }\end{cases}
$$

It is easy to observe that the weights of any two adjacent vertices of $L C_{n}$ are differ by at most 1 . Thus $L C_{n}$ is equitable irregular.

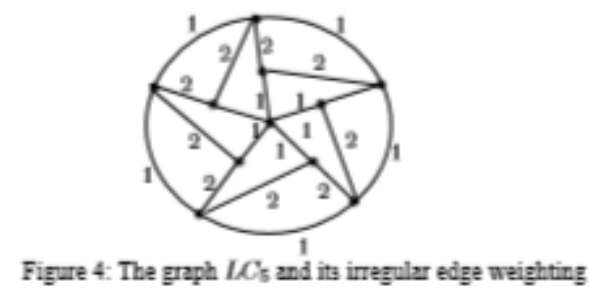

Also, $\mu\left(L C_{n}\right)=3$ and $\Delta\left(L C_{n}\right)=n$, by Theorem 1.1 that $\mathrm{S}_{\mathrm{e}}\left(L C_{n}\right)$ $\geq\left\lfloor\frac{n-1}{2}\right\rfloor$ and the edge-weighting $\varphi$ deduces that $\mathrm{S}_{\mathrm{e}}\left(L C_{n}\right) \leq$ $\left\lfloor\frac{n-1}{2}\right\rfloor$. Hence $\mathrm{S}_{\mathrm{e}}\left(L C_{n}\right)=\left\lfloor\frac{n-1}{2}\right\rfloor$

Definition 2.9. A quadrilateral snake $Q_{n}$ is obtained from a path $u_{1}, u_{2}, \ldots, u_{m}$ by joining $u_{i}$ and $u_{i+1}$ to new vertices $v_{i}$ and $w_{i}$ respectively and joining the vertices $v_{i}$ and $w_{i}$ for $i=1,2, \ldots, n-1$.

Theorem 2.10. The Quadrilateral snake $Q_{n}$ is equitable irregular for all $n$ and $S_{e}\left(Q_{n}\right)=2$.

Proof :Let V $\left(Q_{n}\right)=\left\{u_{1}, u_{2}, u_{3}, \ldots, u_{n+1}\right\} \cup\left\{v_{i} w_{i}\right.$ :

$1 \leq i \leq n-1\}$ and $\mathrm{E}\left(Q_{n}\right)=\left\{u_{i} u_{i+1}: 1 \leq i \leq\right.$ $n\} \cup\left\{u_{i} v_{i}, v_{i} w_{i}, w_{i} u_{i+1}: 1 \leq i \leq n-1\right\}$. Define an edge weighting $\varphi$ of $Q_{n}$ as follows.

Let $\varphi\left(u_{i} u_{i+1}\right)=\varphi\left(w_{i} u_{i+1}\right)=1$ and $\varphi\left(u_{i} v_{i}\right)=1$ for all $2 \leq i \leq n-1$ and assign the label 2 to all the remaining edges of $Q_{n}$. Then the weight of any vertex of $Q_{n}$ is either 3 or 4. 
Therefore, the weights of any two adjacent vertices of $Q_{n}$ differ by at most 1 and so $Q_{n}$ is equitable irregular. (For the graph $Q_{3}$, the equitable irregular edge-weighting $\varphi$ is illustrated in Figure 5). Hence $S_{e}\left(Q_{n}\right) \leq 2$.

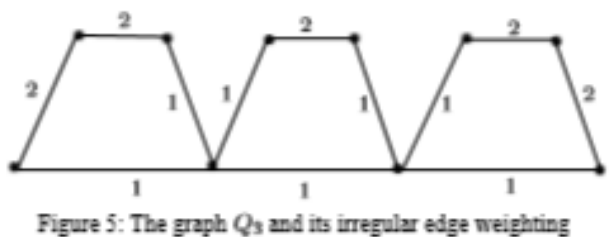

Since $\mu\left(Q_{n}\right)=2$ and $\Delta\left(Q_{n}\right)=4$, it follows by Theorem 1.1, $S_{e}\left(Q_{n}\right) \geq 2$.Thus $S_{e}\left(Q_{n}\right)=2$.

Definition 2.11. A Double Quadrilateral snake $D Q_{n}$ is a graph consists of two quadrilateral snakes that have a common path.

Theorem 2.12. The Double Quadrilateral snake $D Q_{n}$ is equitable irregular for all $n$ and $S_{e}\left(D Q_{n}\right)=4$.

Proof. Let V $\left(D Q_{n}\right)=\left\{u_{1}, u_{2}, u_{3}, \ldots, u_{n+1}\right\} \cup$

$\left\{v_{i}, w_{i}, x_{i}, y_{i}: 1 \leq i \leq n\right\}$ and

$\mathrm{E} \quad\left(D Q_{n}\right.$

$=\left\{u_{i} u_{i+1}, u_{i} v_{i}, v_{i} w_{i}, w_{i} u_{i+1}, u_{i} x_{i}, x_{i} y_{i}, y_{i} u_{i+1}: 1 \leq i \leq\right.$

$n\}$.

Now, let us define an edge weighting $\varphi$ of $D Q_{n}$ as follows. Let $\varphi\left(u_{1} v_{1}\right)=\varphi\left(u_{1} x_{1}\right)=\varphi\left(u_{n+1} w_{n}\right)=\varphi\left(u_{n+1} y_{n}\right)=$ 2 and $\varphi\left(v_{i} w_{i}\right)=\varphi\left(x_{i} y_{i}\right)=4$ for all $1 \leq i \leq n$.

Further, assign the label 1 to all the remaining edges of $D Q_{n}$. Then $S_{\varphi}\left(u_{1}\right)=S_{\varphi}\left(u_{n+1}\right)=5 ; S_{\varphi}\left(v_{1}\right)=S_{\varphi}\left(x_{1}\right)=S_{\varphi}\left(w_{n}\right)=$ $S_{\varphi}\left(y_{n}\right)=6$ and for all $1 \leq i \leq n-1$, we have $S_{\varphi}\left(y_{i}\right)=$ $S_{\varphi}\left(w_{i}\right)=5$ and for all $2 \leq i \leq n, S_{\varphi}\left(u_{i}\right)=6$.

Clearly, the weights of any two adjacent vertices of $D Q_{n}$ are differ by atmost 1 . Therefore $D Q_{n}$ is equitable irregular and hence $S_{e}\left(D Q_{n}\right) \leq 4$.

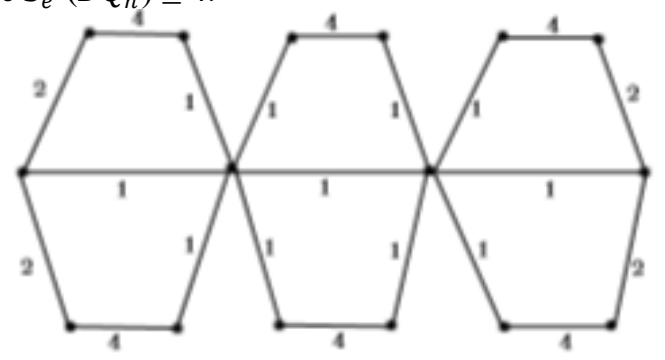

Fipure 6: The graph $D Q 3$ and its imegular edge weighting

Since $\mu\left(D Q_{n}\right)=2$ and $\Delta\left(D Q_{n}\right)=6$, this implies $S_{e}\left(D Q_{n}\right) \geq 4$ by Theorem 1.1. and thus $S_{e}\left(D Q_{n}\right)=4$.

\section{CONCLUSION}

In this paper, wehave proved some special classes of graphs such as closed helm graphs, windmill graphs, flower graphs, quadrilateral snakes and double quadrilateral snakes are equitable irregular.

\section{ACKNOWLEDGMENT}

The authors wish to thank the Management and Head of the Department of mathematics for their Motivation and supportfor this research work.

\section{REFERENCES}

1. G. Chartrand, M. S. Jacobson, J. Lehel, O. R. Oellermann, S. Ruiv and F. Saba, Irregular networks, Congr. Numer.,64 (1988).
2. M. Karonski, T. Luczak and A. Thomson, Edge weights and vertex colours. J. Combinator. Theory B, 91(2004), 151-157.

3. I. Sahul Hamid, and S. Ashok Kumar, Equitable irregular edgeweighting of graphs, SUT Journal of Mathematics, Vol. 46, No. 1 (2010), 79 - 91.

4. J.A.Gallian, A dynamic survey of graph labeling, Electron. J . Comb., 15(2008).

\section{AUTHORS PROFILE}

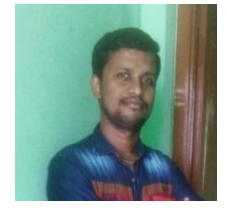

Dr. S. Saravanakumar received his UG, PG and M.Phil Degrees in Mathematics from The Madura College, Madurai in the years 2008, 2010 and 2011 respectively. He obtained his Ph.D. from Madurai Kamaraj University in 2016 and he has published around 6 research articles in reputed SCI and Scopus indexed journals. His current research areas are coloring,decomposition and domination related parameters in graphs. $\mathrm{He}$ is currently working as an Assistant Professor in Mathematics at KalasalingamAcademy of Research and Education, Krishnankoil- 626126, India

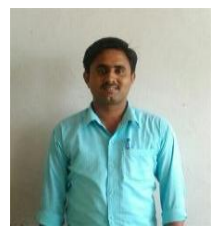

Mr.P.Sankaranarayanan pursued his B.Sc degree from Devangar Arts College, Aruppukottai in 2012.He got his M.Sc degree from S.B.K college, Aruppukottai in 2014. He obtained his M.Phil degree from S.N.College Madurai in 2015. His current research area is Graphs Theory and he is currently working as Assistant Professor in Mathematics atKalasalingam Academy of Research and Education, Anand Nagar, Krishanankoil- 626126, TamilnaduIndia 